\title{
Threshold strategy-proofness: on manipulability in large voting problems
}

Citation for published version (APA):

Ehlers, L. H., Peters, H. J. M., \& Storcken, A. J. A. (2000). Threshold strategy-proofness: on manipulability in large voting problems. METEOR, Maastricht University School of Business and Economics. METEOR Research Memorandum No. 029 https://doi.org/10.26481/umamet.2000029

Document status and date:

Published: 01/01/2000

DOI:

10.26481/umamet.2000029

Document Version:

Publisher's PDF, also known as Version of record

\section{Please check the document version of this publication:}

- A submitted manuscript is the version of the article upon submission and before peer-review. There can be important differences between the submitted version and the official published version of record. People interested in the research are advised to contact the author for the final version of the publication, or visit the DOI to the publisher's website.

- The final author version and the galley proof are versions of the publication after peer review.

- The final published version features the final layout of the paper including the volume, issue and page numbers.

Link to publication

\footnotetext{
General rights rights.

- You may freely distribute the URL identifying the publication in the public portal. please follow below link for the End User Agreement:

www.umlib.nl/taverne-license

Take down policy

If you believe that this document breaches copyright please contact us at:

repository@maastrichtuniversity.nl

providing details and we will investigate your claim.
}

Copyright and moral rights for the publications made accessible in the public portal are retained by the authors and/or other copyright owners and it is a condition of accessing publications that users recognise and abide by the legal requirements associated with these

- Users may download and print one copy of any publication from the public portal for the purpose of private study or research.

- You may not further distribute the material or use it for any profit-making activity or commercial gain

If the publication is distributed under the terms of Article $25 \mathrm{fa}$ of the Dutch Copyright Act, indicated by the "Taverne" license above, 


\title{
Threshold Strategy-Proofness: On Manipulability in Large Voting Problems
}

\author{
Lars Ehlers* \\ Ton Storcken ${ }^{\dagger}$
}

September 2002

\begin{abstract}
In voting problems where agents have well behaved (Lipschitz continuous) utility functions on a multidimensional space of alternatives, a voting rule is threshold strategy-proof if any agent can only obtain a limited utility gain by not voting for a most preferred alternative, given that the number of agents is large enough. For anonymous voting rules it is shown that this condition is not only implied by but in fact equivalent to the influence of any single agent reducing to zero as the number of agents grows. If there are at least five agents, the mean rule (taking the average vote) is shown to be the unique anonymous and unanimous voting rule that meets a lower bound with respect to the number of agents needed to obtain threshold strategy-proofness.
\end{abstract}

JEL Classification numbers: D72, D71.

Keywords: Threshold strategy-proofness, voting problems, mean rule.

Corresponding author: Hans Peters, Department of Quantitative Economics, Faculty of Economics and Business Administration, University of Maastricht, P.O. Box 616, 6200 MD Maastricht, The Netherlands. Fax: +31-43-3883288.

*Département de Sciences Économiques and C.R.D.E., Université de Montréal, Québec H3C 3J7, Canada. E-mail: lars.ehlers@umontreal.ca

$\dagger$ Department of Quantitative Economics, Faculty of Economics and Business Administration, University of Maastricht, P.O. Box 616, 6200 MD Maastricht, The Netherlands. E-mail: h.peters@ke.unimaas.nl, t.storcken@ke.unimaas.nl 


\section{Introduction}

A voting mechanism for public goods is strategy-proof if no voter can gain by not voting according to his true preference. There are good reasons to use strategy-proof voting mechanisms, the main one being that the final decision will be based on the right information. Unfortunately, in general a high price has to be paid in order to attain strategy-proofness. In the classical model of social choice studied by Gibbard (1973) and Satterthwaite (1975) this price is dictatoriality of the mechanism, and in the classical literature on demand revealing public good provision (Clarke, 1971; Groves, 1973) the price is budget imbalance. Better results are obtained if the domains of preferences as well as alternatives are restricted. Moulin (1980) considers single-peaked preferences on the real line and characterizes a class of generalized median mechanisms. However, in a multi-dimensional Euclidean space with singlepeaked preferences, a mechanism is dictatorial if it is strategy-proof and the range of the mechanism is at least two-dimensional (Zhou, 1991). Even in these cases, rather natural mechanisms like taking the average vote, are excluded by strategy-proofness.

In the present paper we question the compellingness of the strategyproofness condition based on the following considerations. First of all, if a mechanism is not strategy-proof, it may yet be difficult and risky for any single agent to try to manipulate the final outcome by insincere voting if he does not exactly know the preferences of the other agents. Moreover, even if he does know these preferences he would have to take into account potential manipulation by the other agents. Second and additional, even if some gain is to be reached by manipulation, this will generally be rather small if there are many agents, and it will not outweigh the cost of finding out the best way to manipulate. For these reasons it seems safe to assume that especially in larger voting problems agents will not be interested in manipulation, even if there is a theoretical potential for gain. Indeed, political scientists do not tend to be particularly interested in strategic aspects of voting systems.

The present paper is an attempt to formalize these considerations. A global summary of our main findings is as follows. In our framework, voting mechanisms that are 'competitive' in the sense that any single voter has negligible influence, are shown to be hardly manipulable. This is obvious and intuitive. More interestingly, for anonymous mechanisms (which is the usual case) also the converse holds: Limited strategic manipulability must imply 
competitiveness of the voting mechanism. Taking the average vote turns out to be the unique anonymous and unanimous mechanism that satisfies limited strategic manipulability in a sharp sense, i.e., for the minimum number of agents.

In somewhat more detail, we will assume a framework where agents have continuous preferences on some Euclidean space, which may represent different aspects of the political spectrum. Limited strategic manipulability is modeled by the condition of threshold strategy-proofness on a voting mechanism or rule. This condition means that, given that there are sufficiently many agents, no agent can gain more than a small amount in utility by not reporting a best point. This unavoidably raises the question how to measure utility gains. This is not possible in a purely ordinal framework, and in fact we will impose a condition of Lipschitz continuity on the utility functions representing the preferences. It should be noted that no single-peakedness condition will be imposed, which makes our model also in this respect different from Moulin (1980) and Zhou (1991).

As announced above, the first main result of the paper is that for anonymous voting rules threshold strategy-proofness is equivalent to a condition saying that, as the number of agents becomes large, the influence of any single agent on the outcome of the voting rule reduces to zero. Next, for a large class of voting rules a sharp lower bound on the number of agents will be derived in order that the maximal utility gain by manipulation is limited. The second main result is that, if there are at least five agents, then anonymity, unanimity, and this sharp lower bound characterize the mean rule (taking the average of the votes). In other words, the number of agents needed to make a voting rule 'almost' (threshold) strategy-proof is minimal in case of the mean rule.

Both results confirm plausible intuitions. The first result not only establishes that strategic manipulation issues vanish if each single voter has a negligible influence, as is the case in large voting problems. More importantly, it says that this negligible influence is a necessary condition in order to avoid strategic manipulation. Note that this result is not obvious: for instance, generalized medians in the one-dimensional case with single-peaked preferences are strategy-proof but do not entail negligable influence of individuals. The second result says that a natural rule like the mean rule seems the best one to use if manipulation biases should only be small.

As far as we are aware the literature on this particular theme is rather 
limited. Gary-Bobo and Jaaidane (2000) show that in a growing consumer population it is possible to have a revelation mechanism for the production of public goods that is strategy-proof, budget balanced, and approximately efficient. The following references concern private goods. Roberts and Postlewaite (1976) show that in an exchange economy the gain from not announcing one's competitive demand goes to zero as the number of consumers increases through replication. Gul and Postlewaite (1992) derive that in an exchange economy with asymmetric information the tension between incentive compatibility and efficiency disappears as the agents are sufficiently replicated. Córdoba and Hammond (1998) show that in a class of smooth random exchange economies there are mechanisms that are nonmanipulable in the limit with probability one. Though in a different (private goods) context, these results are similar in spirit to ours. Schummer (1999) considers a condition of $\epsilon$-dominance of truth-telling in a two-person exchange economy without, however, increasing the number of agents.

Section 2 gives the formal model and a preliminary result. Section 3 characterizes anonymous and threshold strategy-proof voting rules, and Section 4 characterizes the mean rule in terms of sharp threshold strategy-proofness. The more technical proofs are collected in Section 5 .

\section{Preliminaries}

The set of alternatives is the $m$-dimensional Euclidean space $\mathbb{R}^{m}$. A point of $\mathbb{R}^{m}$ can be interpreted as the location of a public decision, or of a political party, in an election with respect to $m$ attributes, or political viewpoints.

Preferences are represented by utility functions $u: \mathbb{R}^{m} \rightarrow \mathbb{R}$ that are Lipschitz continuous, that is, there is an $L>0$ such that $|u(a)-u(b)| \leq$ $L\|a-b\|$ for all $a, b \in \mathbb{R}^{m}$. Here, without loss of generality we take $\|\cdot\|$ to be the Euclidean norm. The number $L$ is called a Lipschitz constant and $\mathcal{U}_{L}$ is the set of all utility functions with Lipschitz constant $L$. Lipschitz continuity in this context can be interpreted, roughly, as the requirement that utility functions do not exhibit fast changes.

For a compact set $C \subseteq \mathbb{R}^{m}$, an alternative $x \in C$ is a best alternative of a utility function $u$ in $C$ if $u(x) \geq u(y)$ for all $y \in C$. Since $u$ is Lipschitz continuous and $C$ compact, a best alternative always exists.

The set of (potential) agents is identified with the set of natural numbers 
$\mathbb{I N}$. Let $\mathcal{P}$ denote the set of all non-empty and finite subsets of $\mathbb{I N}$. For $N \in \mathcal{P},|N|$ denotes the cardinality of the set $N$. A voting problem is a pair $(N, p)$, where $N \in \mathcal{P}$ and $p \in\left(\mathbb{R}^{m}\right)^{N}$ is a profile of votes. This implies that each agent $i \in N$ is allowed to report (vote for) one alternative $p(i) \in \mathbb{R}^{m}$. A voting rule $F$ assigns to each voting problem $(N, p)$ one alternative $F(N, p) \in \mathbb{R}^{m}$. Instead of $F(N, p)$ we will often just write $F(p)$, in particular if it is obvious what the set of agents $N$ is.

For a set of agents $N \in \mathcal{P}$ and an agent $i \in N$, the profiles $p, q$ are called $i$-deviations if $p(j)=q(j)$ for all $j \in N \backslash\{i\}$. The central property under investigation in this paper is the following.

Definition 1 A voting rule $F$ is threshold strategy-proof if for every compact set $C \subseteq \mathbb{R}^{m}$, every $L>0$, and every $\epsilon>0$, there is a real number $k>0$ such that for every $N \in \mathcal{P}$ with $|N| \geq k$, every $i \in N$, all $i$-deviations $p, q \in C^{N}$, and every utility function $u \in \mathcal{U}_{L}$ for which $p(i)$ is a best alternative in $C$, we have:

$$
u(F(q))-u(F(p)) \leq \epsilon .
$$

Threshold strategy-proofness says the following. Suppose that votes are restricted to a compact subset and utility functions are Lipschitz continuous. Then, given that there are sufficiently many agents, an agent cannot gain more than a small amount $\epsilon$ by voting for a possibly suboptimal alternative. In other words, under these conditions it is hardly worthwhile to try manipulation by strategic voting. The usual strategy-proofness condition would require the inequality in the definition to hold for $\epsilon=0$. Hence, threshold strategy-proofness is a relaxation of strategy-proofness.

The following lemma shows an important consequence of threshold strategy-proofness: by manipulation, either the resulting alternative remains approximately the same, or the manipulating agent is almost a dictator with respect to the votes under comparison. This result will be used frequently in the sequel.

Lemma 1 Let $F$ be a threshold strategy-proof voting rule and let $C, L, \epsilon$, and $k$ be as in Definition 1. Let $N \in \mathcal{P}$ be a set of agents with $|N| \geq k$, let $i \in N$, and let $p, q \in C^{N}$ be $i$-deviations. Then $\|F(p)-F(q)\| \leq \epsilon / L$ or $[\|p(i)-F(p)\| \leq \epsilon / L$ and $\|q(i)-F(q)\| \leq \epsilon / L]$. 
Proof. Define $u^{\prime} \in \mathcal{U}_{L}$ by $u^{\prime}(x)=L\|F(p)-x\|$ for all $x \in \mathbb{R}^{m}$. Define $u \in \mathcal{U}_{L}$ by $u(x)=\min \left\{u^{\prime}(x), u^{\prime}(p(i))\right\}$ for all $x \in \mathbb{R}^{m}$. By threshold strategyproofness and the definition of $u$,

$$
\epsilon \geq u(F(q))-u(F(p))=L \min \{\|p(i)-F(p)\|,\|F(p)-F(q)\|\} .
$$

This implies $\|F(p)-F(q)\| \leq \epsilon / L$ or $\|p(i)-F(p)\| \leq \epsilon / L$. The remaining statement in the lemma follows by symmetry.

\section{Anonymity and threshold strategy-proof- ness}

In this section we characterize all anonymous and threshold strategy-proof voting rules. Let $\circ$ denote composition of maps.

Definition 2 A voting rule $F$ is anonymous if for all sets of agents $N, M \in \mathcal{P}$ with $|N|=|M|$, all bijections $\sigma: M \rightarrow N$, and all profiles $p \in\left(\mathbb{R}^{m}\right)^{N}$, we have:

$$
F(N, p)=F(M, p \circ \sigma) .
$$

In other words, a voting rule is anonymous if the identities of the agents do not matter.

Definition 3 A voting rule $F$ is insensitive at large populations if for every $\epsilon>0$ and every compact set $C \subseteq \mathbb{R}^{m}$ there is a real number $k$ such that for every $N \in \mathcal{P}$ with $|N| \geq k$, every $i \in N$, and all $i$-deviations $p, q \in C^{N}$ we have:

$$
\|F(p)-F(q)\| \leq \epsilon .
$$

Thus, if a voting rule is insensitive at large populations, the influence of a single agent becomes small as the number of agents becomes large. Since by Lipschitz continuity small changes in the alternatives can lead to only small changes in utility, the condition implies threshold strategy-proofness. Formally: 
Proposition 1 Let $F$ be a voting rule that is insensitive at large populations. Then $F$ is threshold strategy-proof.

Proof. Let $C$ be a compact subset of $\mathbb{R}^{m}$ and let $\epsilon>0$. Let $L>0$ and choose $k \in \mathbb{R}$ such that for every $N \in \mathcal{P}$ with $|N| \geq k$, every $i \in N$ and all $i$-deviations $p, q \in C^{N}$, we have $\|F(p)-F(q)\| \leq \epsilon / L$. Consequently, for such profiles and for $u \in \mathcal{U}_{L}$, it follows that

$$
u(F(p))-u(F(q)) \leq L\|F(p)-F(q)\| \leq \epsilon .
$$

Hence, $F$ is threshold strategy-proof.

The converse of Proposition 1 does not hold. For instance, a dictatorial rule, assigning to any profile of votes the same agent's reported point, is threshold strategy-proof but not insensitive at large populations. Under anonymity, however, the two conditions are equivalent.

Theorem 1 An anonymous voting rule is threshold strategy-proof if and only if it is insensitive at large populations.

The proof of Theorem 1 is given in Section 5. The if-part (in fact, Proposition 1) states the intuitive and obvious fact that, if the influence of a single agent vanishes as the population of voters becomes large, then so does his potential for strategic manipulation. The only-if part is more interesting and more difficult to prove: if the voting rule is anonymous and the possibility for strategic manipulation vanishes as the population grows, then any single agent's influence must vanish at all. Observe that median rules (Moulin, 1980) are anonymous but not insensitive at large populations: for those rules there always exist situations where a single agent's vote may have a tremendous influence on the public outcome. Consequently, these rules also violate threshold strategy-proofness (that is, outside of the single-peaked domain).

\section{Sharp bounds and the mean rule}

In this section we first derive a lower bound for the number of agents in order that for a given voting rule each agent can gain at most $\epsilon$ by manipulation. In 
other words, we establish a lower bound for the number $k$ as in the definition of threshold strategy-proofness. This will be done for voting rules that are anonymous and translation invariant.

Definition $4 \mathrm{~A}$ voting rule $F$ is translation invariant if for every set of agents $N \in \mathcal{P}$, every $c \in \mathbb{R}^{m}$, and every profile $p \in\left(\mathbb{R}^{m}\right)^{N}$, we have:

$$
F(N, p+(c, \ldots, c))=F(N, p)+c .
$$

Translation invariance makes the voting rule independent of the choice of the zero. It is satisfied by all voting rules of interest. The next lemma provides the announced lower bound. For a compact set $C$ in $\mathbb{R}^{m}$ we define $\operatorname{diam}(C):=\max \{\|a-b\|: a, b \in C\}$.

Lemma 2 Let $F$ be an anonymous, translation invariant and threshold strategy-proof voting rule. Let $C \subseteq \mathbb{R}^{m}$ be convex and compact. Let $\epsilon>0, L>0$, and $k \geq 3$ be as in Definition 1. Then $k \geq L \operatorname{diam}(C) / \epsilon$.

A proof of this lemma is given in Section 5.

The remainder of the section is devoted to the study of voting rules that meet the lower bound derived in Lemma 2. This will result in a characterization of the mean rule. First, we formalize this condition.

Definition 5 A voting rule $F$ is sharp threshold strategy-proof if for every compact subset $C \subseteq \mathbb{R}^{m}$, every $L>0$, every $\epsilon>0$, every $N \in \mathcal{P}$ with $|N| \geq L \operatorname{diam}(C) / \epsilon$, all $i$-deviations $p, q \in C^{N}$, and all utility functions $u \in \mathcal{U}_{L}$ for which $p(i)$ is a best alternative on $C$, we have:

$$
u(F(q))-u(F(p)) \leq \epsilon .
$$

An example of a sharp threshold strategy-proof voting rule is the mean rule $F^{\text {mean }}$, defined by $F^{\text {mean }}(p)=\sum_{i \in N} p(i) /|N|$ for every $N \in \mathcal{P}$ and every profile $p \in\left(\mathbb{R}^{m}\right)^{N}$. Sharp threshold strategy-proofness follows from the fact that for $p, q$ as in Definition 5,

$$
\begin{aligned}
u\left(F^{\text {mean }}(q)\right)-u\left(F^{\text {mean }}(p)\right) & \leq L\left\|F^{\text {mean }}(p)-F^{\text {mean }}(q)\right\| \\
& =L\|p(i)-q(i)\| /|N| \\
& \leq L \operatorname{diam}(C) /|N| \\
& \leq \epsilon .
\end{aligned}
$$


It turns out that, basically, the mean rule is the unique sharp threshold strategy-proof voting rule of interest. The following theorem makes this statement precise. A voting rule $F$ is unanimous if $F(a, \ldots, a)=a$ for every $a \in \mathbb{R}^{m}$.

Theorem 2 Let $F$ be a unanimous, anonymous and sharp threshold strategyproof voting rule. Then $F$ is equal to the mean rule for all profiles with at least five agents.

A proof of this theorem can be found in Section 5 .

Theorem 2 does not hold for less than four agents. Let $m=1$ and let $F$ be the mean rule in case the number of agents is four or more. For three agents, let $F$ assign to every profile $p$ the point $\frac{2}{3} \min \{p(i): i \in N\}+\frac{1}{3} \max \{p(i)$ : $i \in N\}$; and for two agents let $F$ assign the lower one of the reported votes. Altogether, this defines a voting rule satisfying the properties in Theorem 2 . It is an open problem whether the rule can also be different for the border line case of four agents. An answer to this problem, however, is of limited interest since we are concerned with large voting problems.

The following examples establish the logical independence of the properties in Theorem 2. In these examples, $m=1$.

Example 1 For all $N \in \mathcal{P}$ and $p \in \mathbb{R}^{N}$, let

$$
\tilde{F}(p):=\operatorname{median}(\min \{p(i): i \in N\}, 0, \max \{p(i): i \in N\}) .
$$

This rule is unanimous and anonymous, but not threshold strategy-proof. $\triangleleft$

Example 2 For all $N \in \mathcal{P}$ and $p \in \mathbb{R}^{N}$, let $F^{0}(p):=0$. This rule is anonymous and sharp threshold strategy-proof, but not unanimous.

Example 3 For all $N \in \mathcal{P}$ and $p \in \mathbb{R}^{N}$, let $F^{\prime}(p):=p(\min N)$. This rule is unanimous and sharp threshold strategy-proof, but not anonymous.

\section{Proofs}

We start with a technical, auxiliary lemma. For $a \in \mathbb{R}^{m}$ and $\epsilon>0$ denote by $B(a, \epsilon):=\left\{x \in \mathbb{R}^{m}:\|a-x\| \leq \epsilon\right\}$ the ball with centre $a$ and radius $\epsilon$. For a set $C \subseteq \mathbb{R}^{m}, \operatorname{conv}(C)$ denotes the convex hull of $C$, i.e., the smallest convex set containing $C$. 
Lemma 3 Let $a, b, c \in \mathbb{R}^{m}$ such that $c=\frac{1}{2}(a+b)$. Let $\lambda>0$ such that $\|a-b\| \geq 5 \lambda$. Let $C^{1}=\operatorname{conv}\left(\{a\} \cup B\left(c, \frac{1}{2} \lambda\right)\right), C^{2}=\operatorname{conv}\left(\{b\} \cup B\left(c, \frac{1}{2} \lambda\right)\right)$, and $C=\operatorname{conv}\left(C^{1} \cup C^{2}\right)\left(=C^{1} \cup C^{2}\right)$.

Let $F$ be a voting rule and let $N \in \mathcal{P}$ and assume that for all $i \in N$ and all $i$-deviations $p, q \in C^{N}$

$$
\|F(p)-F(q)\| \leq \lambda \text { or }[\|F(p)-p(i)\| \leq \lambda \text { and }\|F(q)-q(i)\| \leq \lambda]
$$

with all inequalities strict whenever $p, q \in\left(C^{1}\right)^{N}$ or $p, q \in\left(C^{2}\right)^{N}$.

Let $r \in B\left(c, \frac{1}{2} \lambda\right)^{N}$ and $j \in N$, and for $x \in \mathbb{R}^{m}$ denote by $r^{x}$ the $j$ deviation of $r$ defined by $r(j)=x$. Assume that $\left\|F\left(r^{a}\right)-a\right\| \leq \lambda$ and $\left\|F\left(r^{b}\right)-b\right\| \leq \lambda$.

Then $F$ is not anonymous.

Proof. Without loss of generality let $N=\{1, \ldots, n\}$ and $j=n$.

For $t \in\{0,1, \ldots, n-1\}$ and $x \in \mathbb{R}^{m}$ let $w^{t, x}$ be the profile defined for all $i \in N$ by

$$
w^{t, x}(i)= \begin{cases}r^{x} & \text { if } i>t \\ c & \text { if } i \leq t .\end{cases}
$$

For $t \in\{0,1, \ldots, n-1\}$ we now prove that

$$
\left\|F\left(w^{t, a}\right)-a\right\| \leq \lambda \text { and }\left\|F\left(w^{t, b}\right)-b\right\| \leq \lambda .
$$

Case $t=0$. Since $w^{0, a}=r^{a}$ and $w^{0, b}=b$ this is the assumption made in the statement of the lemma.

Case $t=1$. Since $w^{1, a}, w^{0, a}$ are 1-deviations, (1) implies

$$
\left\|F\left(w^{1, a}\right)-F\left(w^{0, a}\right)\right\|<\lambda \text { or }\left\|F\left(w^{0, a}\right)-r^{a}(1)\right\|,\left\|F\left(w^{1, a}\right)-c\right\|<\lambda .
$$

Since by case $t=0$ we have $\left\|F\left(w^{0, a}\right)-a\right\| \leq \lambda$ and, further, $r^{a}(1) \in B\left(c, \frac{1}{2} \lambda\right)$ and $\|a-c\| \geq \frac{5}{2} \lambda$, we have $\left\|F\left(w^{0, a}\right)-r^{a}(1)\right\| \geq \lambda$. So (3) implies $\| F\left(w^{1, a}\right)-$ $F\left(w^{0, a}\right) \|<\lambda$. Since $\left\|F\left(w^{0, a}\right)-a\right\| \leq \lambda$, it follows that $\left\|F\left(w^{1, a}\right)-a\right\|<2 \lambda$. Similarly, $\| F\left(w^{1, b}-b \|<2 \lambda\right.$.

Since $w^{1, a}, w^{1, b}$ are $n$-deviations in $C^{N},(1)$ implies

$$
\left\|F\left(w^{1, a}\right)-F\left(w^{1, b}\right)\right\| \leq \lambda \text { or }\left\|F\left(w^{1, a}\right)-a\right\|,\left\|F\left(w^{1, b}\right)-b\right\| \leq \lambda .
$$


As $\| F\left(w^{1, a}-a\|<2 \lambda\| F,\left(w^{1, b}-b \|<2 \lambda\right.\right.$, and $\|a-b\| \geq 5 \lambda$, the first inequality in (4) does not hold. Hence (4) implies $\left\|F\left(w^{1, a}\right)-a\right\| \leq \lambda$ and $\left\|F\left(w^{1, b}\right)-b\right\| \leq \lambda$. This concludes case $t=1$.

Cases $t=2, \ldots, n-1$ are analogous to case $t=1$. Hence, we have proved (2).

For $x, y \in C$ consider the profile $v^{x, y} \in C^{N}$ defined for all $i \in N$ by

$$
v^{x, y}(i)= \begin{cases}x & \text { if } i=1 \\ c & \text { if } 1<i<n \\ y & \text { if } i=n .\end{cases}
$$

Since $v^{b, a}$ and $w^{n-1, a}$ are 1-deviations in $C^{N}$ we have by (1) that $\| F\left(v^{b, a}\right)-$ $F\left(w^{n-1, a}\right) \| \leq \lambda$ or $\left[\left\|F\left(v^{b, a}\right)-b\right\| \leq \lambda\right.$ and $\left.\left\|F\left(w^{n-1, a}\right)-c\right\| \leq \lambda\right]$. Now the latter cannot be the case since $\|a-c\| \geq \frac{5}{2} \lambda$ and, by (2) for $t=n-$ 1, $\left\|f\left(w^{n-1, a}\right)-a\right\| \leq \lambda$. Hence the former must be the case, $\| F\left(v^{b, a}\right)-$ $F\left(w^{n-1, a}\right) \| \leq \lambda$, and together with $\left\|f\left(w^{n-1, a}\right)-a\right\| \leq \lambda$ this implies $\| F\left(v^{b, a}\right)-$ $a \| \leq 2 \lambda$.

Similarly one proves $\left\|F\left(v^{a, b}\right)-b\right\| \leq 2 \lambda$. Since $\|a-b\| \geq 5 \lambda$, it follows that $F\left(v^{a, b}\right) \neq F\left(v^{b, a}\right)$. So $F$ is not anonymous.

Proof of Theorem 1. In view of Proposition 1 it is sufficient to prove the only-if part. Suppose that $F$ is threshold strategy-proof but not insensitive at large populations. Then it is sufficient to prove that $F$ is not anonymous.

Since $F$ is not insensitive at large populations there is a $\delta>0$ and a compact subset $C^{\prime}$ of $\mathbb{R}^{m}$ such that for every $k^{\prime}>0$ there is a set of agents $N^{\prime}$ with $\left|N^{\prime}\right| \geq k^{\prime}$ and a $j \in N^{\prime}$ and $j$-deviations $v, w \in\left(C^{\prime}\right)^{N^{\prime}}$ with

$$
\|F(v)-F(w)\| \geq \delta .
$$

Take $c \in C^{\prime}, a, b \in \mathbb{R}^{m}$, and $\lambda>0$ such that $c=\frac{1}{2}(a+b), C^{\prime} \subseteq B\left(c, \frac{1}{2} \lambda\right)$, and $\|a-b\| \geq 5 \lambda$. Let $C, C^{1}, C^{2}$ as in Lemma 3 .

Take $0<\epsilon<\min \left\{\lambda, \frac{1}{2} \delta\right\}$. By Lemma 1 there is a $k>0$ such that for all $N \in \mathcal{P}$ with $|N| \geq k$, all $i \in N$ and all $i$-deviations $p, q \in C^{N}$ we have:

$$
\|F(p)-p(i)\|,\|F(q)-q(i)\| \leq \epsilon<\lambda \text { or }\|F(p)-F(q)\| \leq \epsilon<\lambda .
$$

Take $k^{\prime}$ (as in the second paragraph of the proof) equal to $k$, and let $N^{\prime}, j$, $v$, and $w$ as in the second paragraph. Note that (1) in Lemma 3 is satisfied. So by this lemma, letting $v$ play the role of $r$, it is sufficient to prove that

$$
\left\|F\left(v^{a}\right)-a\right\| \leq \lambda \text { and }\left\|F\left(v^{b}\right)-b\right\| \leq \lambda,
$$


where, analogously to $r^{x}$ in Lemma $3, v^{x}$ is the $j$-deviation of $v$ with $v(j)=x$. By (6) we have:

$$
\left\|F\left(v^{a}\right)-F(v)\right\| \leq \epsilon \text { or }\left\|F\left(v^{a}\right)-a\right\| \leq \epsilon
$$

and

$$
\left\|F\left(w^{a}\right)-F(w)\right\| \leq \epsilon \text { or }\left\|F\left(w^{a}\right)-a\right\| \leq \epsilon .
$$

Suppose that $\left\|F\left(v^{a}\right)-a\right\|>\lambda$. Then $\left\|F\left(v^{a}\right)-a\right\|>\epsilon$ and since $v^{a}=w^{a}$, we have both $\left\|F\left(v^{a}\right)-F(v)\right\| \leq \epsilon$ and $\left\|F\left(w^{a}\right)-F(w)\right\|=\left\|F\left(v^{a}\right)-F(w)\right\| \leq \epsilon$. This implies $\|F(v)-F(w)\| \leq 2 \epsilon<\delta$, in contradiction with (5). Thus, we must have $\left\|F\left(v^{a}\right)-a\right\| \leq \lambda$. Similarly, one proves $\left\|F\left(v^{b}\right)-b\right\| \leq \lambda$. This completes the proof of the theorem.

We proceed with the proof of Lemma 2. For a compact set $C \subseteq\left(\mathbb{R}^{m}\right)^{N}$, points $a$ and $b$ in $C$ are called diametrical if $\operatorname{diam}(C)=\|a-b\|$.

Proof of Lemma 2. Let $a$ and $b$ be diametrical points in $C$. Without loss of generality suppose $k \in \mathbb{N}$. Let $N=\{1,2, \ldots, k\}$ and let the profiles $p, q \in C^{N}$ be defined by

$$
p(i)=a+(i-1)(b-a) / k \text { and } q(i)=a+i(b-a) / k \text { for every } i \in N .
$$

Since $C$ is convex, for all $i \in N, p(i), q(i) \in C$. By translation invariance, $F(q)=F(p)+(b-a) / k$. Since $k \geq 3$, we have $\|F(p)-a\| \geq\|b-a\| / k$ or $\|F(q)-b\| \geq\|b-a\| / k$. Without loss of generality assume $\|F(p)-a\| \geq$ $\|b-a\| / k$. Let $u^{\prime} \in \mathcal{U}_{L}$ be defined by $u^{\prime}(x)=L\|F(p)-x\|$ for all $x \in \mathbb{R}^{m}$, and $u$ by $u(x)=\min \left\{u^{\prime}(x), u^{\prime}(a)\right\}$. (This construction is similar as in the proof of Lemma 1.) Note that, by anonymity, $p$ and $q$ can be regarded as 1-deviations. We have

$$
\begin{aligned}
\epsilon & \geq u(F(q))-u(F(p)) \\
& =u(F(p)+(b-a) / k) \\
& =\min \{L\|b-a\| / k, L\|F(p)-a\|\} \\
& =L\|b-a\| / k
\end{aligned}
$$

where the inequality follows from threshold strategy-proofness, the first equality from $F(p)=F(q)+(b-a) / k$ and $u(F(p))=0$, and the third equality 


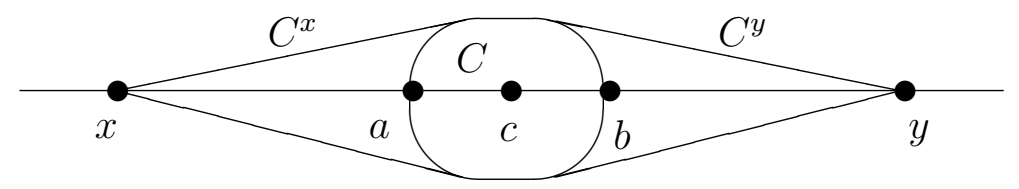

Figure 1: Proof of Lemma 4

from $\|F(p)-a\| \geq\|b-a\| / k$. Hence, $k \geq L\|b-a\| / \epsilon$, which was to be proved.

The proof of Theorem 2 is based on the following auxiliary lemma.

Lemma 4 Let $F$ be an anonymous and sharp threshold strategy-proof voting rule. Let $N \in \mathcal{P}$ with $n=|N| \geq 5$. Let $C$ be a compact and convex subset of $\mathbb{R}^{m}$ with diametrical points $a$ and $b$. Let $j \in N$ and let $p, q \in C^{N}$ be $j$ deviations such that $p(j)=a$ and $q(j)=b$. Then $\|F(p)-F(q)\| \leq\|b-a\| / n$.

Proof. Suppose, to the contrary, that

$$
\|F(p)-F(q)\|>\|b-a\| / n .
$$

Take $L>0$ and $\epsilon=L\|a-b\| / n$. Let $c=\frac{1}{2}(a+b)$ and for $x \in \mathbb{R}^{m}$ let $p^{x}$ be the $j$-deviation of $p$ defined by $p^{x}(j)=x$. For $x, y \in \mathbb{R}^{m}$ such that $a \in \operatorname{conv}(\{x, b\})$ and $b \in \operatorname{conv}(\{y, a\})$ let $C^{x}=\operatorname{conv}(\{x\} \cup C)$ and $C^{y}=$ $\operatorname{conv}(\{y\} \cup C)$. (Cf. Figure 1.) Then $\operatorname{diam}\left(C^{x}\right)=\|x-b\|$ and $\operatorname{diam}\left(C^{y}\right)=$ $\|y-a\|$.

By applying sharp threshold strategy-proofness and Lemma 1 (take for $\epsilon$ in Lemma 1 the number $L\|b-x\| / n$ ) we have for all $i$-deviations $v, w \in\left(C^{x}\right)^{N}$ :

$$
\begin{aligned}
& \|F(v)-F(w)\| \leq\|b-x\| / n \text { or } \\
& \|F(v)-v(i)\|,\|F(w)-w(i)\| \leq\|b-x\| / n .
\end{aligned}
$$

Similarly, for all $i$-deviations $v, w \in\left(C^{y}\right)^{N}$ :

$$
\begin{aligned}
& \|F(v)-F(w)\| \leq\|a-y\| / n \text { or } \\
& \|F(v)-v(i)\|,\|F(w)-w(i)\| \leq\|a-y\| / n .
\end{aligned}
$$

For $v=p$ and $w=q$ and noting that $p(j)=a$ and $q(j)=b$, we have by $(7)$, (8) applied for $x=a$, and (9) applied for $y=b$ :

$$
\|F(p)-a\|,\|F(q)-b\| \leq\|a-b\| / n \text {. }
$$


Since $n \geq 5,(10)$ implies

$$
\|F(p)-F(q)\| \geq 3\|a-b\| / n .
$$

We now first prove the following claim for $x$ and $y$ as above.

Claim $\left\|F\left(p^{x}\right)-x\right\| \leq\|b-x\| / n$ and $\left\|F\left(p^{y}\right)-y\right\| \leq\|a-y\| / n$.

Proof of Claim. Take a sequence $z_{0}=a, z_{1}, \ldots, z_{t}=x$ in $\operatorname{conv}(\{x, a\})$ such that $\left\|z_{\ell}-z_{\ell-1}\right\|<\frac{1}{2}\left\|z_{\ell-1}-b\right\|$ for all $\ell=1, \ldots, t$. We prove that $\left\|F\left(p^{z_{\ell}}\right)-z_{\ell}\right\| \leq\left\|b-z_{\ell}\right\| / n$ for all $\ell=1, \ldots, t$, from which the first inequality in the Claim follows. The second inequality can be proved analogously.

Case $i=1$. First observe that by (11)

$$
\begin{aligned}
& \left\|F\left(p^{z_{0}}\right)-F\left(p^{z_{1}}\right)\right\|+\left\|F\left(p^{z_{1}}\right)-F\left(p^{b}\right)\right\| \\
= & \left.\left\|F(p)-F\left(p^{z_{1}}\right)\right\|+\| F\left(p^{z_{1}}\right)-F(q)\right) \| \\
\geq & \|F(p)-F(q)\| \\
\geq & 3\|a-b\| / n \\
> & 2\left\|z_{1}-b\right\| .
\end{aligned}
$$

Apply (8) on $C^{z_{1}}$ to the pair $p^{z_{1}}$ and $p^{b}=q$. If $\left\|F\left(p^{z_{1}}\right)-F(q)\right\|>\left\|b-z_{1}\right\| / n$ then $\left\|F\left(p^{z_{1}}\right)-z_{1}\right\| \leq\left\|b-z_{1}\right\| / n$ and we are done. If $\left\|F\left(p^{z_{1}}\right)-F(q)\right\| \leq$ $\left\|b-z_{1}\right\| / n$ then by $(12)\left\|F(p)-F\left(p^{z_{1}}\right)\right\|>\left\|b-z_{1}\right\| / n$, hence (8) applied to the pair $p, p^{z_{1}}$ on $C^{z_{1}}$ implies $\left\|F\left(p^{z_{1}}\right)-z_{1}\right\| \leq\left\|b-z_{1}\right\| / n$ and we are again done.

Furthermore, by (10) we have $\|F(q)-b\| \leq\|a-b\| / n \leq\left\|z_{1}-b\right\| / n$, so since $n \geq 5$ we have

$$
\left\|F\left(p^{z_{1}}\right)-F(q)\right\| \geq 3\left\|b-z_{1}\right\| / n .
$$

Hence,

Cases $\ell=2, \ldots, t$ can be proved analogously to case $\ell=1$.

Now take $\lambda>0$ and $\hat{a}, \hat{b}, c \in \mathbb{R}^{m}$ such that $c=\frac{1}{2}(\hat{a}+\hat{b}),\|\hat{a}-\hat{b}\|=5 \lambda$, $C \subseteq B\left(c, \frac{1}{2} \lambda\right), C^{1}=\operatorname{conv}\left(\{\hat{a}\} \cup B\left(c, \frac{1}{2} \lambda\right)\right), C^{2}=\operatorname{conv}\left(\{\hat{b}\} \cup B\left(c, \frac{1}{2} \lambda\right)\right)$. (See Figure 2.) Then, by the Claim, $\left\|F\left(p^{\hat{a}}\right)-\hat{a}\right\| \leq \operatorname{diam}\left(C^{1}\right) / n<\lambda$ and $\| F\left(p^{\hat{b}}\right)-$ $\hat{b} \| \leq \operatorname{diam}\left(C^{2}\right) / n<\lambda$. Furthermore, Lemma 1 and sharp threshold strategyproofness imply that for all $i$-deviations $v, w$ in $\left(C^{k}\right)^{N}$, where $k \in\{1,2,3\}$ and $C^{3}=C^{1} \cup C^{2}$, we have

$$
\|F(v)-F(w)\| \leq \operatorname{diam}\left(C^{k}\right) / n \leq \lambda
$$




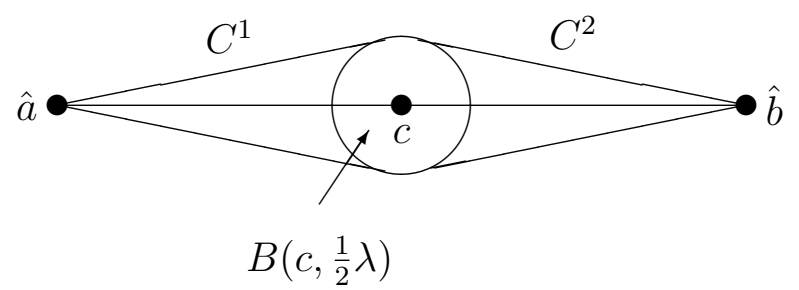

Figure 2: Proof of Lemma 4

or

$$
\|F(v)-v(j)\|,\|F(w)-w(j)\| \leq \operatorname{diam}\left(C^{k}\right) / n \leq \lambda .
$$

Note that the inequalities $\operatorname{diam}\left(C^{k}\right) / n \leq \lambda$ are strict for $k=1,2$. Hence, Lemma 3 applies and yields a contradiction.

Proof of Theorem 2. Let $N \in \mathcal{P}$ have at least five agents and let $p \in$ $\left(\mathbb{R}^{m}\right)^{N}$ be a profile. We proceed by induction on $|p(N)|$, where $p(N)=$ $\{p(i): i \in N\}$.

If $|p(N)|=1$, then $F(p)=F^{\text {mean }}(p)$ by unanimity.

For the induction step, let $|p(N)|=k>1$. Let $C$ be the convex hull of $p(N)$. Take $a$ and $b$ in $p(N)$ diametrical in $C$. Let $S=\{i \in N: p(i) \notin\{a, b\}\}$. Let $|S|=s$ and $n=|N|=k+l+s$ such that $p=\left(a^{k}, p_{S}, b^{l}\right)$. Here, $a^{k}$ means that $k$ agents vote for $a$, and $p_{S}=(p(i))_{i \in S}$. Let $q=\left(a^{k+l}, p_{S}\right)$ and $r=\left(p_{S}, b^{k+l}\right)$. The induction hypothesis entails

$$
F(q)=F^{\text {mean }}(q)=\frac{k+l}{n} a+\frac{s}{n} F^{\text {mean }}\left(p_{S}\right)
$$

and

$$
F(r)=F^{\text {mean }}(r)=\frac{k+l}{n} b+\frac{s}{n} F^{\text {mean }}\left(p_{S}\right) .
$$

It follows that

$$
\|F(q)-F(r)\|=\frac{k+l}{n}\|b-a\| .
$$

By repeated application of Lemma 4,

$$
\|F(q)-F(p)\| \leq \frac{l}{n}\|b-a\|
$$


and

$$
\|F(r)-F(p)\| \leq \frac{k}{n}\|b-a\| .
$$

It follows that these inequalities must be equalities, and in particular

$$
\begin{aligned}
F(p) & =\frac{k}{k+l} F(q)+\frac{l}{k+l} F(r) \\
& =\frac{k}{n} a+\frac{l}{n} b+\frac{s}{n} F^{\text {mean }}\left(p_{S}\right) \\
& =F^{\text {mean }}(p) .
\end{aligned}
$$

This completes the proof of the induction step and of the theorem.

\section{References}

Clarke, E. (1971): "Multipart Pricing of Public Goods," Public Choice, 11, $17-33$.

Córdoba, J.M., and P.J. Hammond (1998): "Asymptotically Strategy-Proof Walrasian Exchange," Mathematical Social Sciences, 36, 185-212.

Gary-Bobo, R.J., and T. Touria Jaaidane (2000): "Polling Mechanisms and the Demand Revelation Problem," Journal of Public Economics, 76, 203238.

Gibbard, A. (1973): "Manipulation of Voting Schemes: A General Result," Econometrica, 41, 587-601.

Groves, T. (1973): "Incentives in Teams," Econometrica, 41, 617-631.

Gul, F., and A. Postlewaite (1992): "Asymptotic Efficiency in Large Exchange Economies with Asymmetric Information," Econometrica, 60, 12731292.

Moulin, H. (1980): "On Strategy-Proofness and Single Peakedness," Public Choice, 35, 437-455.

Roberts, D.J., and A. Postlewaite (1976): "The Incentives for Price-Taking 
Behavior in Large Exchange Economies," Econometrica, 44, 115-127.

Satterthwaite, M. (1975): "Strategy-Proofness and Arrow's Conditions: Existence and Correspondence Theorem for Voting Procedures and Social Choice Functions," Journal of Economic Theory, 10, 187-217.

Schummer, J. (1999): "Almost-Dominant Strategy Implementation," Working Paper, Northwestern University.

Zhou, L. (1991): "Impossibility of Strategy-Proof Mechanisms in Economies with Pure Public Goods," Review of Economic Studies, 58, 107-119. 